\title{
Automatic Highly Accurate Estimation of Gaussian Noise Level in Digital Images Using Filtration and Edges Detection Methods
}

\author{
Serhiy V. Balovsyak \\ Yuriy Fedkovych Chernivtsi National University, Chernivtsi, Ukraine \\ Email: s.balovsyak@chnu.edu.ua \\ Khrystyna S. Odaiska \\ Yuriy Fedkovych Chernivtsi National University, Chernivtsi, Ukraine \\ Email:k.odaiska@chnu.edu.ua
}

Received: 08 October 2017; Accepted: 08 November 2017; Published: 08 December 2017

\begin{abstract}
In this paper we propose a highly accurate method of automatically estimation of the Gaussian noise level in digital images, which is based on image filtering and analysis of the region of interest. Noise level is an important parameter to many digital image processing applications, for example, when removing noise. As the noise level its standard deviation is calculated. The selection of the noise component in an image is performed by high-pass filtration, where the Laplacian difference is used as the filter kernel. Based on the noise component of the image, regions of interest with homogeneous areas of the image are calculated. Region of interest are selected by the iterative method using lowpass filtration, where Gaussian two-dimensional function is used as the filter kernel. The noise level is calculated only in the regions of interest that contain almost no edges and textures, because edges and textures cause errors during the noise level estimation. In order to improve the accuracy of the method, edges of images are detected and out of region of interest. The high accuracy of the proposed method provides the use of high-pass and low-pass filtrations, iterative selection of region of interest and analysis of image edges. The accuracy of the developed method has been tested on the processing of 100 test images with different levels of software added Gaussian noise, as well as the processing of real photos with noise. The proposed method for the noise level estimation can be used for optimal automatic image filtering and for assessing the quality of photosensitive sensors.
\end{abstract}

Index Terms - Noise level estimation, Gaussian noise, digital image filtering, region of interest, image edges.

\section{INTRODUCTION}

Noise level is an important parameter for many digital image processing applications: noise removal, contrast improvement, edge detection, segmentation, pattern recognition etc. [1-3]. If there is noise, high quality digital image processing is impossible without information on its level. In general, the noise level in the image is unknown, so accurate calculation of noise level is necessary to effectively execute the application image processing tasks. The noise level calculated for images acquired from photosensitive sensors can be used to assess the quality of such sensors [1]. Modern methods of calculating the noise level in the images do not always provide the required precision and automatic processing, so the development of a highly accurate and automatic noise level estimation method is an important application problem.

We will consider the noise level for the noise described by the widespread Additive White Gaussian Noise (AWGN) model [1, p.149-161, 2. p.336]. The noise in the AWGN model will be further simplified to Gaussian noise (or normal noise). The level of Gaussian noise is described by its standard deviation $\sigma_{N}$.

Methods of the noise level estimation in the images are divided into manual and automatic. Manual techniques, for example, are based on the manual selection of the region of interest (ROI) in the image [3, p.153-158], show high accuracy, but are laborious. There are promising automatic methods for the noise level estimation in the images for software implementation. They are divided into:

1. Filter-based approaches [4-5], in which noise images are processed by high-pass or low-pass filters using a convolution. The noise level is calculated on the basis of the filtered image for high-pass filters or the difference between the original and filtered images for low-pass filters. The main problem of these methods is the dependence of the filtering result not only on noise, but also on the signal, especially for images with clear edges and rich textures.

2. Patch-based approaches or block-based methods [4], in which the image is divided into homogeneous blocks. Within the blocks, the noise level is calculated, for example, by the method of principal component analysis 
(PCA). The main problem of block algorithms is optimal division of an image into homogeneous blocks.

3. Statistical approaches (SA) [6] are based on filtration of images and calculation of kurtosis values, which depends on the noise level. The accuracy of the methods decreases with the presence of textures in the images.

4. Methods that use Wavelet transform [5] and provide an analysis of wavelet coefficients with a certain threshold. One of the problems of such methods is the optimal choice of threshold value.

5. Methods based on the Fourier analysis of image spectra that take into account the difference between the signal spectrum and noise spectrum [3]. The use of such methods is appropriate if the spectra of the valid signal and noise are significantly different.

Each of these methods has certain restrictions and disadvantages, which stimulate the development of new methods for noise level estimation in the images. In this paper, the filter-based method of noise level estimation is used due to its high accuracy and speed of operation [4]. The disadvantage of the filter-based method, namely the reduction of its accuracy for images with clear edges and rich textures, is eliminated by calculating the image noise level only in region of interest. The iterative selection of region of interest is performed taking into account the change of standard deviation of Gaussian noise, which occurs as a result of image filtering. The region of interest should not contain the edges of the image, therefore, the edges are additionally calculated by Sobel method for more accurate selection of the region of interest. The software implementation of the proposed method for noise level calculation is performed in MATLAB [3].

\section{Mathematical Model of GAussian Noise LeVEL ESTIMATION BY FILTER-BASED METHOD}

The original digital image $f_{n}$, for which it is necessary to estimate the experimental noise level $\sigma_{N E}$, is written into a rectangular matrix $f_{n}=\left(f_{n}(i, k)\right)$, where $i=1, \ldots, M$, $k=1, \ldots, N$ (Fig. 1a, Fig. 1b) [3]. All images are processed in the shades of gray. The intensity $f_{n}$ is normalized within a range from 0 to 1 .

In the model AWGN the probability density function (PDF) for noise is described by the following formula [2, p. 336]:

$$
P_{D F}(z)=\frac{1}{\sqrt{2 \pi} \sigma_{N}} \exp \left(\frac{-\left(z-z_{C}\right)^{2}}{2 \sigma_{N}^{2}}\right)
$$

where $z$-value of image brightness, $z_{C}$ - mean (average) value of $z, \sigma_{N}$ - theoretical standard deviation of noise (noise level). The squared standard deviation of noise is called a dispersion (variance) $D_{N}=\sigma_{N}^{2}$.
To select the noise component $f_{h}$ (Fig. 1c), in the image $f_{n}$, a high-pass spatial filtration $[2,3]$ is carried out by method of convolution of $f_{n}$ with a high-pass filter kernel $w_{H}=\left(w_{H}(m, n)\right)$ of $M_{w} \times N_{w}$ elements by the expression

$$
f_{h}(i, k)=\sum_{m=1}^{M_{w}} \sum_{n=1}^{N_{w}} f_{n}(i-m, k-n) \cdot w_{H}(m, n),
$$

where $f_{h}=\left(f_{h}(i, k)\right)$ - image of the noise component (of the same size as $\left.f_{n}\right) ; i=1, \ldots, M, k=1, \ldots, N$.

The convolution of the image $f_{n}$ with a kernel $w_{H}$ is simplified to

$$
f_{h}=f_{n} * w_{H}
$$

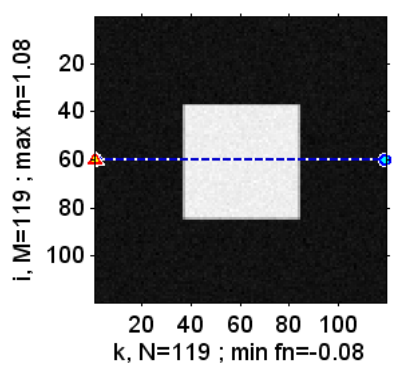

(a)

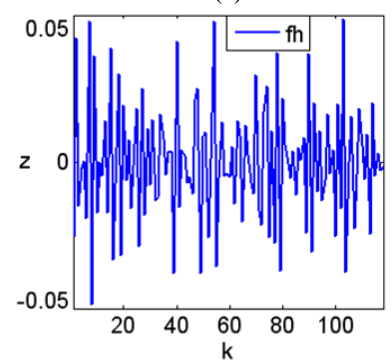

(c)

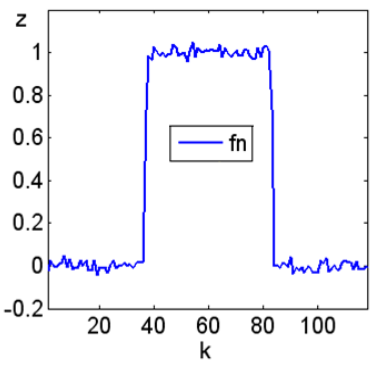

(b)

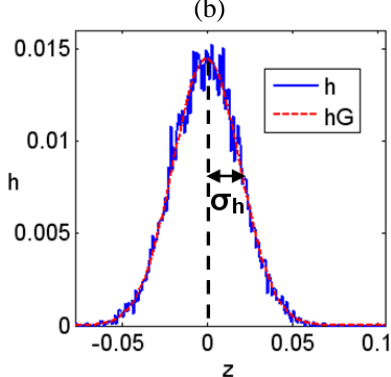

(d)

Fig.1. Example of Gaussian noise level estimation by the filter-based method in the image with software added Gaussian noise with theoretical noise level $\sigma_{N}=2 \%$ : a) original image $f_{n}$, the markers indicate the beginning and end of the profile (Fig. 1b); b) original image profile $\left.f_{n} ; \mathrm{c}\right)$ image profile of the noise component $f_{h} ;$ d) histogram $h(z)$ of the image $f_{h}, \sigma_{h}=0.0197, Q_{h}=256, h_{G}$ is the Gaussian function with standard deviation $\sigma_{h}$, which approximates the histogram $h(z)$

As a high-pass filter kernel, the Laplacian kernel is applied, the value of elements is generally calculated by the following expression [7]:

$$
w_{H a}=\frac{4}{(a+1)}\left(\begin{array}{ccc}
\frac{a}{2} & \frac{1-a}{4} & \frac{a}{2} \\
\frac{1-a}{4} & -1 & \frac{1-a}{4} \\
\frac{a}{2} & \frac{1-a}{4} & \frac{a}{2}
\end{array}\right) \text {, }
$$

where $a$-Laplacian filter parameter, $0 \leq a \leq 1$.

For $a=0$ from (4) we obtain the kernel $w_{H 0}$ of the Laplacian filter 


$$
w_{H 0}=\left(\begin{array}{ccc}
0 & 1 & 0 \\
1 & -4 & 1 \\
0 & 1 & 0
\end{array}\right),
$$

and for $a=1-$ the kernel $w_{H 1}$ of the Laplacian filter

$$
w_{H 1}=\frac{1}{2}\left(\begin{array}{ccc}
1 & 0 & 1 \\
0 & -4 & 0 \\
1 & 0 & 1
\end{array}\right)
$$

The high-pass filter should emphasize brightness differences for all directions [8-10], so values of the elements of the resulting kernel $w_{H}$ of the high-pass filter are calculated as the difference between kernels $w_{H 1}$ and $w_{H 0}$ by the formula [6]:

$$
w_{H}=\frac{2}{6}\left(w_{H 1}-w_{H 0}\right)=\frac{1}{6}\left(\begin{array}{ccc}
1 & -2 & 1 \\
-2 & 4 & -2 \\
1 & -2 & 1
\end{array}\right) \text {. }
$$

The noise component image $f_{h}$ received as the result of convolution of $f_{n}$ with the kernel $w_{H}$ (7) practically does not depend on the edges (Fig. 1c). For further estimation of the experimental noise level $\sigma_{N E}$ the standard deviation $\sigma_{h}$ of histogram $h(z)$ (Fig. 1d) of the image $f_{h}$ is used. The brightness $z$ of the histogram $h(z)$ is divided into $Q_{h}$ subintervals.

There is Gaussian noise with standard deviation $\sigma_{N}$ in the original image $f_{n}$, so the brightness of each pixel $f_{n}$ is a random variable with a dispersion $D_{N}=\sigma_{N}^{2}$. Taking into account the properties of the convolution (2), the dispersion of the noise $D_{N H}=D\left(f_{h}(i, k)\right)$ of an arbitrary pixel of noise component image $f_{h}$ is equal to the arithmetic mean $M_{w} \times N_{w}$ of equally distributed and mutually independent random variables $f_{n}\left(i_{1}, k_{1}\right)$ multiplied by the corresponding elements of the kernel $w_{H}(m, n)$ :

$$
\begin{aligned}
& D_{N H}=D\left(\sum_{m=1}^{M_{w}} \sum_{n=1}^{N_{w}} f_{n}\left(i_{1}, k_{1}\right) \cdot w_{H}(m, n)\right)= \\
& =D_{N} \sum_{m=1}^{M_{w}} \sum_{n=1}^{N_{w}} w_{H}^{2}(m, n),
\end{aligned}
$$

where $D_{N}$ is the dispersion of each of the random variables $f_{n}\left(i_{1}, k_{1}\right), D_{N}=D\left(f_{n}\left(i_{1}, k_{1}\right)\right) ; \quad i_{1}=i-m, k_{1}=k$ $n$. The properties of the dispersion are used in the (8): the constant factor can be put outside dispersion sign and squared; the dispersion of the sum of independent variables is equal to the sum of the components dispersion [11].

Standard deviation of Gaussian noise $\sigma_{N H}$ for the image $f_{h}$ is calculated by its dispersion (8) according to the formula:

$$
\sigma_{N H}=\sqrt{D_{N H}}=\sigma_{N} \sqrt{\sum_{m=1}^{M_{w}} \sum_{n=1}^{N_{w}} w_{H}^{2}(m, n)}
$$

The coefficients of the kernel $w_{H}(7)$ are chosen in such a way that according to (9) $\sigma_{N H}=\sigma_{N}$. Since the value of the standard deviation of Gaussian noise $\sigma_{N H}$ in the image $f_{h}$ is equal to the standard deviation $\sigma_{h}$ of its histogram (Fig. 1d), therefore, taking into account (9) the experimental value of noise level $\sigma_{N E}(\%)$, in the first approximation is equal to:

$$
\sigma_{N E}=100 \sigma_{N}
$$

The above-mentioned calculation sequence of the noise level $\sigma_{N E}$ is described for the entire image processing case, but can be used to process the selected areas of image.

\section{Proposed Algorithm AND SOFTWARE For ESTIMATION OF GAUSSIAN NOISE LEVEL}

Using the mathematical models of the Gaussian noise level estimation given above the algorithm and corresponding software have been developed in MATLAB system.

When calculating the noise level based on the entire image of the edges and textures cause a significant error, that's why to improve the accuracy of the method, it is necessary to remove the edges and textures from the region of interest. The precise calculation of the ROI implies the spatial average processing of the noise component in the image $f_{h}$, so the $f_{d}$ image is initially calculated as the absolute value of $f_{h}$ :

$$
f_{d}=\left|f_{h}\right|
$$

After this, the convolution of the image $f_{d}$ with the Gaussian low-pass filter kernel with standard deviation $\sigma_{\mathrm{wL}}$ is carried out. As the result the averaged image of noise level $f_{d c}$ is received (Fig. 2a)

$$
f_{d c}=f_{d} * w_{L}
$$

The edges and textures correspond to a high level $f_{d}$, as well as $f_{d c}$, therefore the ROI contains only the pixels of image $f_{h}$, for which the values of the corresponding pixels $f_{d c}$ do not exceed the set threshold $T_{h}$ (Fig. 2c). For pixels of images that belong to the ROI, " 1 " is written into the matrix $f_{R O I}$ according to the formula:

$$
f_{R O I}(i, k)=\left\{\begin{array}{l}
1, f_{d c}(i, k) \leq T_{h} \\
0, f_{d c}(i, k)>T_{h}
\end{array},\right.
$$

where $i=1, \ldots, M, k=1, \ldots, N, T_{h}-$ threshold of ROI. 
After calculating the ROI, the standard deviation value $\sigma_{h}$ of the image $f_{h}$ is calculated only for the pixels that belong to the ROI (Fig. 2b).

Threshold $T_{h}$ of the ROI is calculated as

$$
T_{h}=z_{C}+k_{T h} \cdot \sigma_{f d c}
$$

where $z_{C}$ is the mean value of $f_{d c}$ (Fig. 2c); $\sigma_{\mathrm{fdc}}-$ standard deviation of $f_{d c} ; k_{T h}$ - threshold coefficient.

The mean value $z_{c}$ of the image $f_{d c}$ is calculated as the standard deviation [11] of the brightness of the image $f_{h}$ by the formula:

$$
z_{C}=\sqrt{\frac{2}{\pi}} \sigma_{h}=0.798 \sigma_{h}
$$

The standard deviation value $\sigma_{\mathrm{fdc}}$ of the image $f_{d c}(12)$ is calculated by the formula:

$$
\sigma_{f d c}=\sigma_{h} \sqrt{\sum_{m=1}^{M_{w}} \sum_{n=1}^{N_{w}} w_{L}^{2}(m, n)},
$$

is similar to the (9) with the replacement of the unknown standard deviation value of the noise $\sigma_{N}$ with its approximation $\sigma_{h}$.

The coefficient $k_{T h}$ are chosen so that in the image $f_{d c}$ the values of the most pixels (where noise prevails) were lower than the threshold $T_{h}(14)$. The approximate value of the coefficient $k_{T h}=3$, because in this case almost all Gaussian noise values (> 99.7\%) [10] will be lower than the threshold $T_{h}$ according to the $3 \sigma$ rule.

When calculating the ROI on the basis of the threshold $T_{h}$, a significant number of pixels belonging to edges is removed from it. However, some of the edges pixels that remain in the ROI may lead to an error in the calculation of the experimental noise level. Therefore, the proposed method for calculating the noise level provides two modes for the ROI selection:

1. ROI selection mode based on only threshold $T_{h}$ (mode \#1).

2. ROI selection mode is initially based on the threshold $T_{h}$, and then the ROI correction with the edges is taken into account (mode \#2). In this mode, image edges are calculated by the Sobel method with the threshold $T_{s}$ [2, p.187-190]. The received edges are removed from the ROI, which increases the accuracy of the noise level estimation.

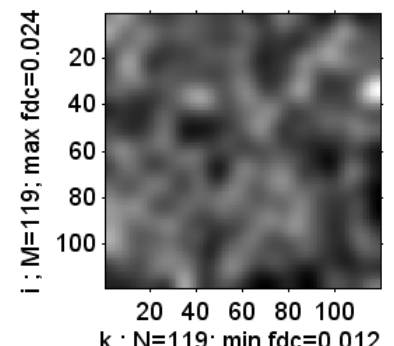

(a)

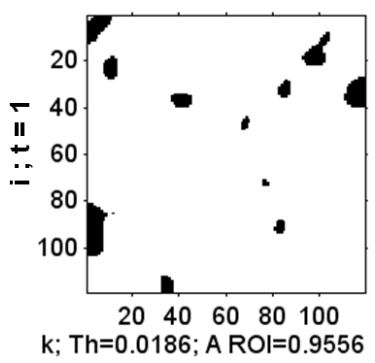

(b)

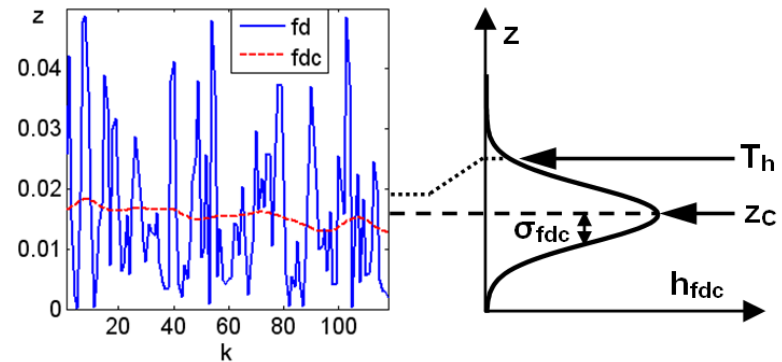

(c)

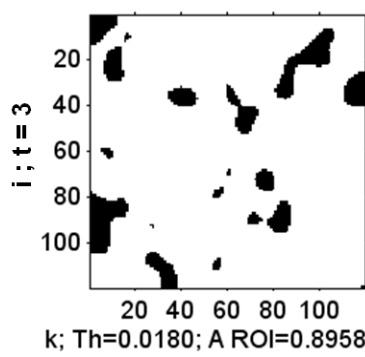

(d)

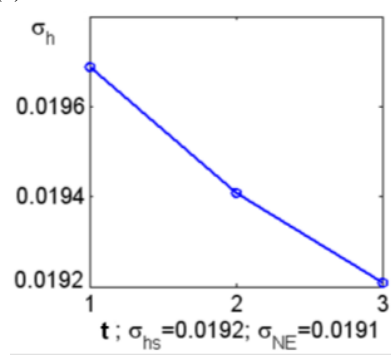

(e)
Fig.2. Example of Gaussian noise level estimation in the image (Fig. 1a) by the proposed method: a) image $f_{d c}$; b) $f_{R O I}$ image of the ROI (ROI contains white pixels) for iteration $t=1, A_{R O I}$ - relative area of the ROI; c) image profiles of $f_{d}$ and averaged image of noise level $f_{d c}$ for $t=1$, initial $\sigma_{h}=0.0201$ (for the whole image), $\sigma_{f d c}=0.0013, z_{C}=0.0160-$ mean value of $f_{d c}, T_{h}=0.0186$ - threshold of $f_{d c}$, standard deviation of low-pass Gaussian kernel $\sigma_{w L}=4.5$; d) image $f_{R O I}$ of the ROI for iteration $t=3$; e) graph of iterative correction $\sigma_{h}(t)$, the experimental noise level $\sigma_{N E}=1.91 \%$

The Gaussian noise level was calculated for low and high pass valid signal images [12], to which Gaussian noise was software added (simulated) with the theoretical noise level $\sigma_{N}$. Taking into account the results of the obtained calculations the precise value of the standard deviation of the Gaussian filter kernel $\sigma_{w L}$ is determined, and also specified the formulae for calculating the threshold $T_{h}$ and the experimental noise level $\sigma_{N E}$.

The results of the research have shown that the minimum mean square error of the calculation of experimental noise level $\sigma_{N E}$ (in reference to $\sigma_{N}$ ) is obtained for the following parameters:

1. The standard deviation value of the kernel $w_{L}$ of the Gaussian filter $\sigma_{w L}=4.5$, which is used for convolution $f_{d c}=f_{d} * w_{L}$ (Fig. 2a).

2. The threshold $T_{h}$ of the ROI is calculated for the threshold coefficient $k_{T h}=2.02$ and the standard deviation of Gaussian filter kernel $\sigma_{w L}=4.5$ by the (14), which takes into account (15) and (16) into the form

$$
T_{h}=0.798 \sigma_{h}+2.02 \cdot 0.063 \sigma_{h}=0.925 \sigma_{h} .
$$

3. The experimental noise level $\sigma_{N E}(\%)$ in the mode \#2 of ROI selection (taking into account the edges of the image) is calculated using the standard deviation $\sigma_{h}$ according to the refined equation (10):

$$
\sigma_{N E}=\left(100 \sigma_{h}-\sigma_{\min 2}\right)^{k_{\sigma h 2}},
$$


where the coefficients $\sigma_{\min 2}=0.02$ and $k_{\mathrm{ch} 2}=1.01$ take into account the fact that standard deviation $\sigma_{h}$ in the image $f_{h}$ increases more slowly than the noise level (since at large $\sigma_{h}$, the ROI does not usually include all pixels with the predominant noise component).

4. The experimental noise level $\sigma_{N E}(\%)$ in the mode \#1 of ROI selection (excluding image edges) is calculated using the standard deviation $\sigma_{h}$ according to the refined equation (10):

$$
\sigma_{N E}=\left(100 \sigma_{h}-\sigma_{\min 1}\right)^{k} \sigma h 1,
$$

where the coefficient $\sigma_{\min 1}=0.03$.

In this case, according to the empirical formula (19), the calculated experimental noise levels $\sigma_{N E}$ at large values of standard deviation $\sigma_{h}\left(\sigma_{h} \approx 0.2\right)$ are lower than the theoretical noise levels $\sigma_{N}$. Therefore, for a more accurate calculation of $\sigma_{N E}$, an adaptive coefficient $k_{\text {бh } 1}$ is used which is calculated as a sigmoid function with constant component

$$
k_{\sigma h 1}=k_{\sigma h 0}+\frac{\Delta_{\sigma h}}{1+\exp \left(-\left(\sigma_{h}-m_{\sigma h}\right)\right)},
$$

where the constant component $k_{\text {oh } 0}=1.0125$, coefficients $\Delta_{\mathrm{\sigma h}}=0.008, m_{\mathrm{\sigma h}}=0.18$; the expression (20) for the adaptive coefficient $k_{\text {oh } 1}$ provides the increase $\sigma_{N E}$ only for the necessary range of $\sigma_{h}$ (Fig. 3).

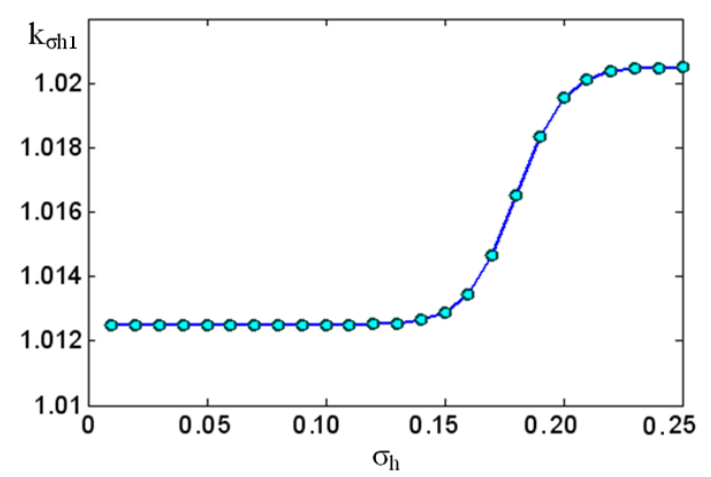

Fig.3. The graph of the sigmoid function (20), which describes the dependence $k_{\text {。hl }}\left(\sigma_{h}\right)$

The algorithm of the proposed method for estimating the level of Gaussian noise in images is as follows (Fig. 4). After reading of the initial image $f_{n}$, kernels $w_{H}$ and $w_{L}$ are created, respectively, for high-pass and lowpass filters. On the basis of $f_{n}$, the noise component image $f_{h}$ and its module $f_{d}$ are calculated by (3) and (11), respectively. The averaged image of the noise level $f_{d c}$ is calculated as a convolution of the image $f_{d}$ with the Gaussian low-pass filter $w_{L}$ (12). On the image of the noise component $f_{h}$ the standard deviation $\sigma_{h}$ of its histogram is calculated. For an image $f_{n}$, the edges of $g_{c}$ are calculated by the Sobel method which can be used to specify the ROI. Then there is a process of specifying the ROI and its corresponding standard deviation $\sigma_{h}$ (for image $f_{h}$ ) in the cycle with the counter $t$. For each iteration $t$ the threshold $T_{h}(17)$ is calculated.

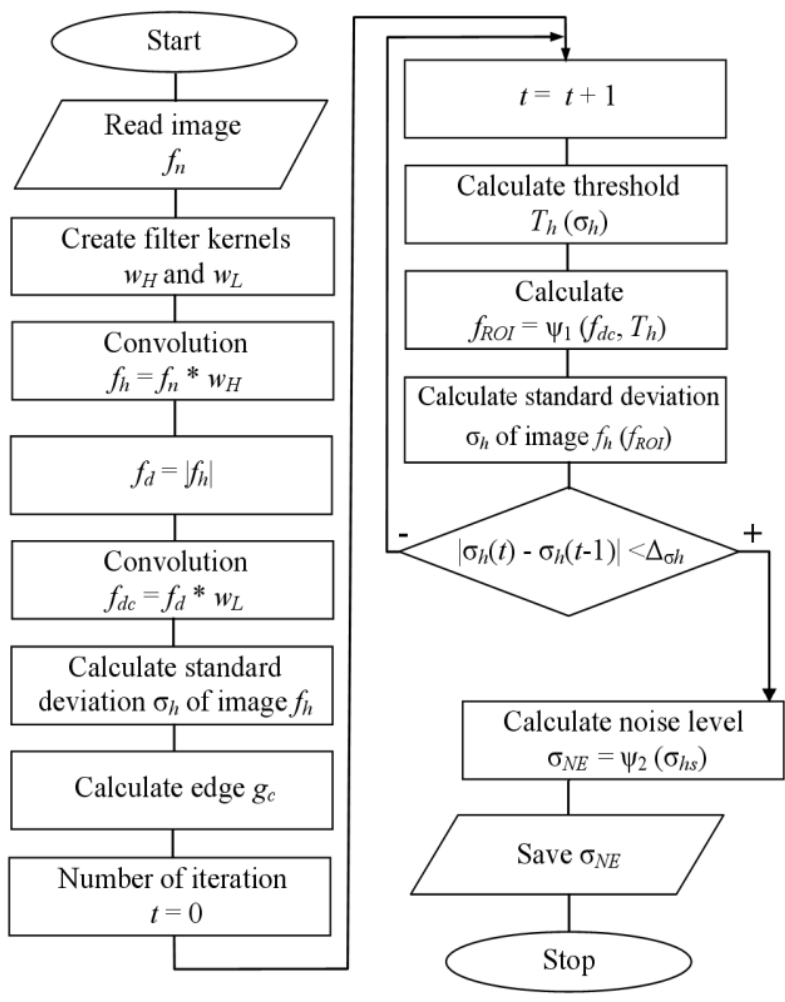

Fig.4. Scheme of the proposed algorithm for Gaussian noise level estimation in images

Based on the threshold $T_{h}$ and the averaged image of the noise level $f_{d c}$, the ROI is calculated where the function $\psi_{1}$ is described by the formula (13). If the $\sigma_{h}$ change for the iteration $t$ in reference to the previous value $\sigma_{h}(t-1)$ does not exceed the constant $\Delta_{\text {oh }}$ (for example, $\left.\Delta_{\mathrm{\sigma h}}=0.004\right)$, the refinement process $\sigma_{h}$ is completed. On the basis of the refined value $\sigma_{h s}$, the experimental value of noise level $\sigma_{N E}$ is calculated where the function $\psi_{2}$ is described by the (18) for the mode \#2 of ROI selection and by (19) for the mode \#1 of ROI selection.

According to the described algorithm (Fig. 4), the refined image of the ROI (Fig. 2d) for the original image $f_{n}$ (Fig. 1a) is obtained, as well as the corresponding values $\sigma_{h}$ and $\sigma_{N E}$ (Fig. 2e). Since the homogeneous areas dominate in the initial image (Fig. 1a), the ROI refinement is insignificant (Fig. 2). The experimental noise level $\sigma_{N E}$ is close to the theoretical $\sigma_{N}$ (the difference is $0.09 \%$ ).

\section{Testing of Proposed Method for IMAges With ADDED NOISE}

The proposed method HLROI (High-pass \& Low-pass filtration \& Region Of Interest) of the Gaussian noise level estimation based on high-pass filtration, low-pass filtration and the selection of the region of interest has been tested during the calculation of experimental noise level $\sigma_{N E}$ for a set of images.

Consider first examples of noise level estimation in mode \#1 of ROI selection (without taking into account image edges). 
The proposed method provides high accuracy of noise level estimation even for images with rich textures (Fig. 5, Fig. 6). At the same time, practically only homogeneous regions are selected for the theoretical noise level $\sigma_{N}=2 \%$ (Fig. 5a) in the regions of interest, where Gaussian noise prevails (Fig. 5c, Fig. 5d). Texture areas are not the ROI parts, which ensures high accuracy of calculation of the experimental noise level $\sigma_{N E}$ (error $0.09 \%$ ). The proposed method has high convergence, so that the ROI and correspondingly the noise level value $\sigma_{N E}$, are specified for just 7 iterations (Fig. 5b).

When processing images with a high theoretical noise level $\sigma_{N}=12 \%$ (Fig. 6a), not only homogeneous areas are selected in the regions of interest, but also areas with textures where Gaussian noise prevails (Fig. 6c, Fig. 6d). At the same time, areas with rich textures are not included in the ROI, which ensures high accuracy of the calculation of the experimental noise level $\sigma_{N E}$ (error $0.11 \%$ ). For high levels of noise and textures in the image, the convergence of the developed method slightly increases (Fig. 6b), making the ROI more precise with the permissible quantity of iterations. High convergence of the method ensures its speed at program implementation.

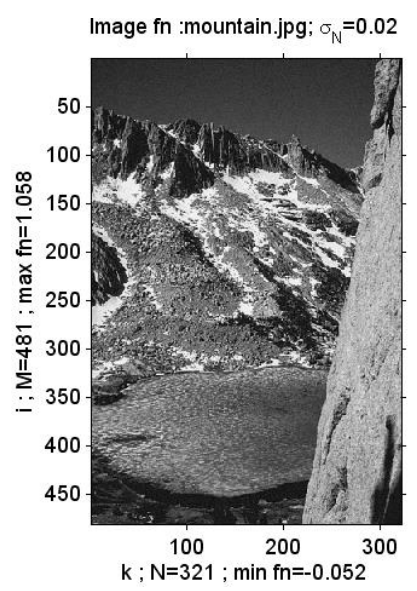

(a)

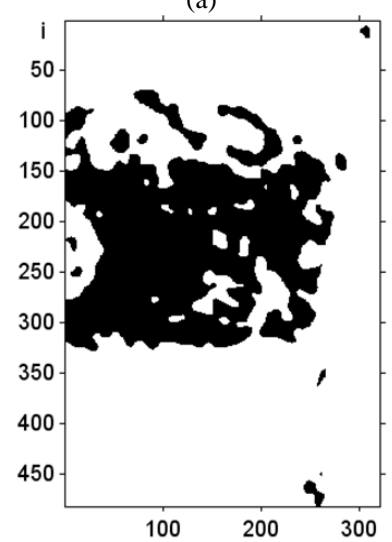

$\mathrm{k} ; \mathrm{Th}=0.0571 ; \mathrm{A}$ ROI=0.7230; $\mathrm{t}=1$

(c)

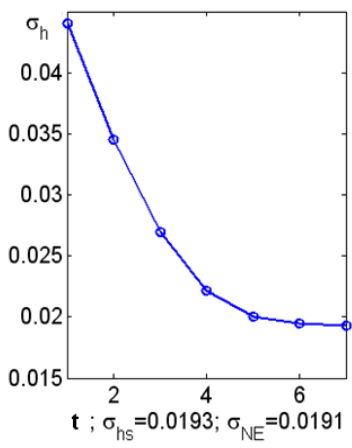

(b)

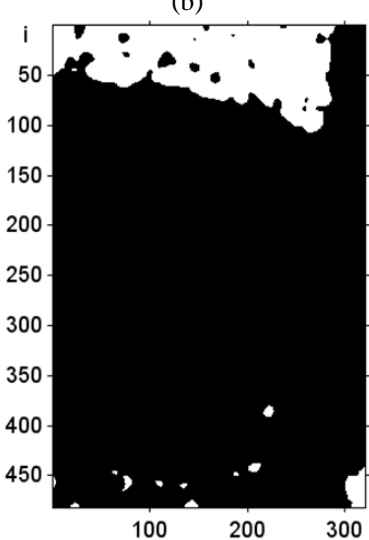

$k ;$ Th=0.0180; A ROI=0.1247; $t=7$

(d)
Fig.5. Example of the Gaussian noise level estimation by the proposed HLROI method in the image "Mountain" $[13,14]$ with textures

$\left(\sigma_{N}=2 \%\right)$ : a) the original image $\left.f_{n} ; \mathrm{b}\right)$ graph of iterative refinement $\sigma_{h}$ $(t)$, the experimental noise level $\sigma_{N E}=1.91 \%$; c) image of the ROI for iteration $t=1$; d) image of the ROI for iteration $t=8$

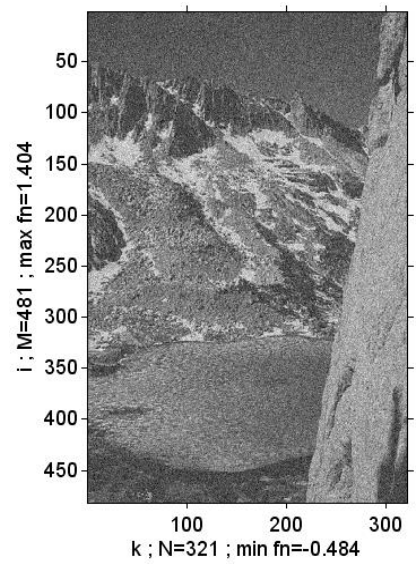

(a)

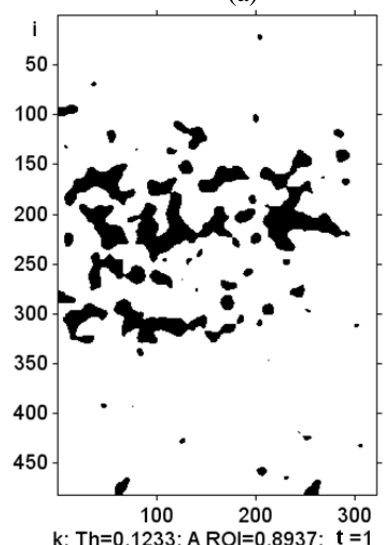

(c)

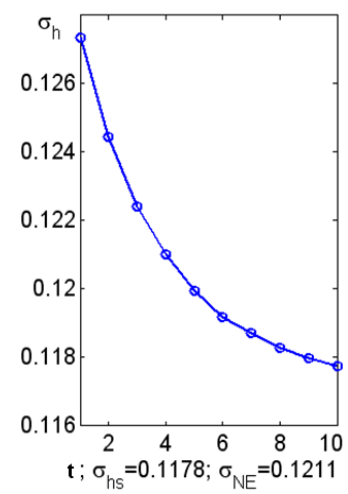

(b)

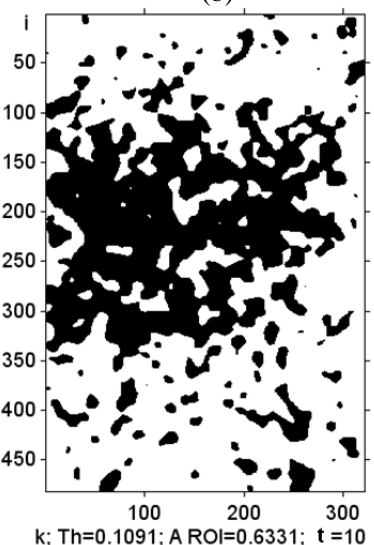

(d)

Fig.6. Example of the Gaussian noise level estimation by the proposed HLROI method in the image "Mountain" $[13,14]$ with textures $\left(\sigma_{N}=12 \%\right)$ : a) the original image $f_{n} ;$ b) graph of iterative refinement $\sigma_{h}$ $(t)$, the experimental noise level $\sigma_{N E}=12.11 \%$; c) image of the ROI for iteration $\mathrm{t}=1 ; \mathrm{d}$ ) image of the ROI for iteration $t=10$

For the classical test images with low, medium and large level of details (Fig. 7), high accuracy of the experimental noise level $\sigma_{N E}$ is achieved by the proposed method (Fig. 8), which is practically the same as the theoretical noise level $\sigma_{N}$.

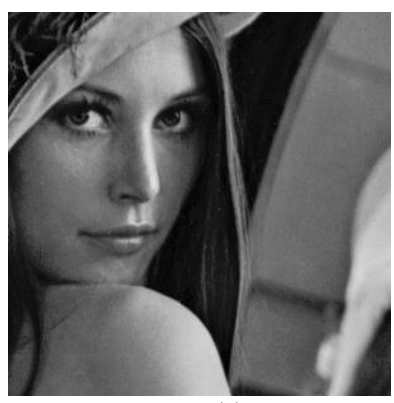

(a)

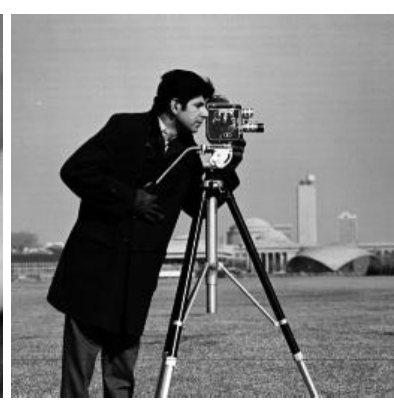

(b) 


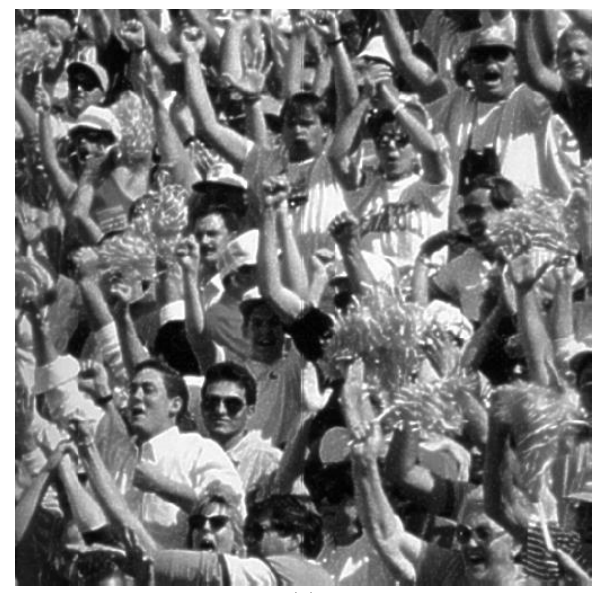

(c)

Fig.7. Test images with low, medium and large level of details [12]: a) image «Face» with a low level of details, $300 \times 300$ pixels size;

b) image «Cameraman» with a medium level of details, $256 \times 256$ pixels size; c) image «Crowd» with a large level of details $512 \times 512$ pixels size

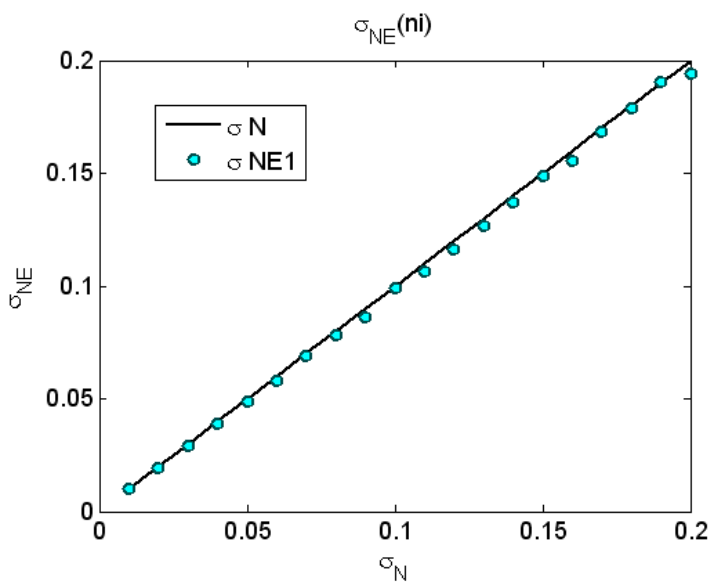

(a)

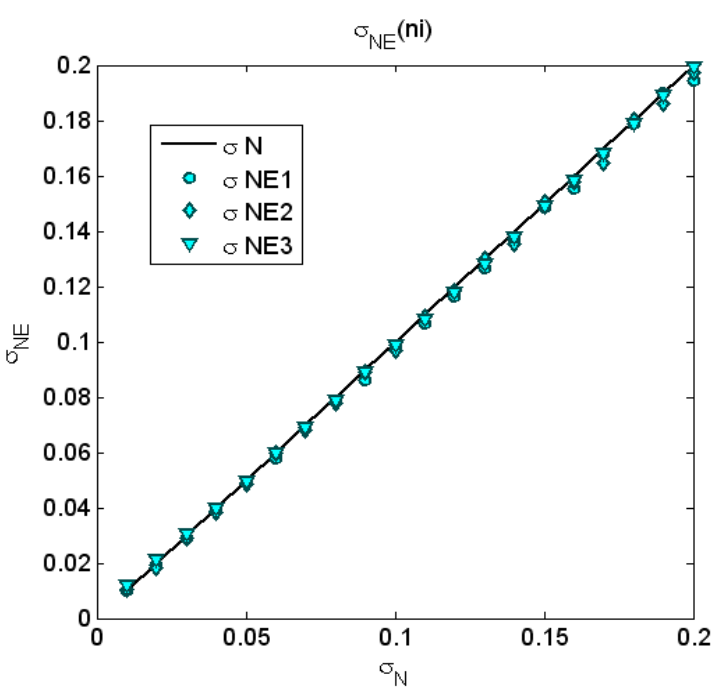

(b)

Fig. 8. Calculation of the experimental level $\sigma_{N E}$ of Gaussian noise by the proposed HLROI method in images with low, medium and large level of details (Fig. 7) and theoretical noise level $\sigma_{N}$ : a) experimental noise levels for the image «Face» (Fig 7a); b) experimental noise levels for images «Face» $(\sigma \mathrm{NE} 1)$, «Cameraman» $(\sigma \mathrm{NE} 2)$, «Crowd» $(\sigma \mathrm{NE} 3)$; RMSES $=0.212 \%$ is the root of mean square error (RMSE), which is calculated between the values $\sigma_{N E}$ and $\sigma_{N}$ for all images
When calculating the noise level for images with clear edges (Fig. 9a), the accuracy of the $\sigma_{N E}$ estimation can be increased (especially for $\sigma_{N}<5 \%$ ) by Sobel edge detection and removing edges from the ROI (Fig. 9). Such image processing is realized in the mode \#2 of ROI selection (taking into account the edges of the image). Due to the removal of edges (Fig. 9b) from the ROI, the threshold $T_{h}$ value is calculated correctly, therefore the experimental value of the noise level $\sigma_{N E}$ is calculated with a slight error of $0.26 \%$ (Fig. 9d). In the case of calculating the noise level for the same image (Fig. 9a) by the proposed method in the mode \#1 of ROI selection (without taking into account the edges of the image) clear edges lead to higher values of threshold $T_{h}$. As a result, significant set of edges remains in the ROI, resulting in the higher value of the experimental noise level $\sigma_{N E}$ is calculated with an error of $2.71 \%$.

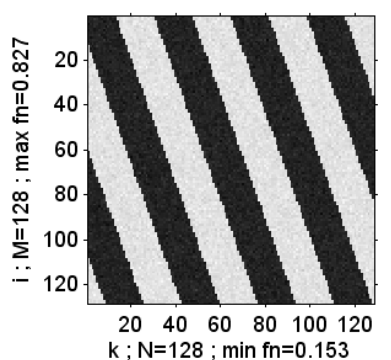

(a)

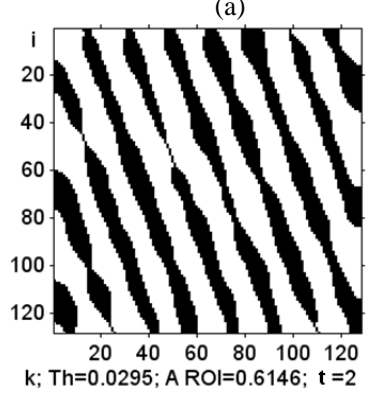

(c)

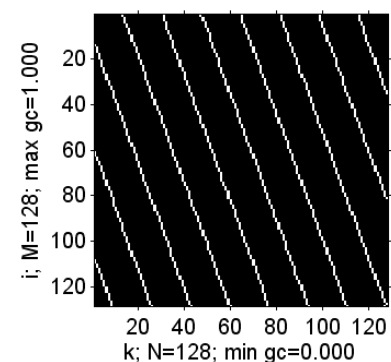

(b)

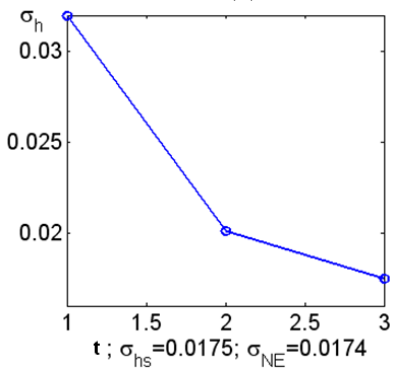

(d)
Fig.9. Example of the Gaussian noise level estimation by the proposed method, taking into account the edges in the image of the bands, with theoretical noise level $\sigma_{N}=2 \%$ : a) original image $f_{n}$; b) image of the edges $g_{c}$, calculated by the Sobel method with the threshold $T_{s}=0.08$; c) image of the ROI for iteration $t=2$; d) graph of the iterative refinement $\sigma_{h}(t)$, the experimental noise level $\sigma_{N E}=1.74 \%$

The results of the estimation of the experimental Gaussian noise level $\sigma_{N E}$ for the test set (100 images) of the Berkeley image database BSDS300 [13, 14] (Fig. 10) by the proposed HLROI method show its high accuracy in both selection modes of ROI (Fig. 11, Table 1). For most images that do not contain a significant high-pass component of a signal (clear edges and rich textures [15, $16])$, the value of experimental noise level $\sigma_{N E}$ calculated by the proposed method almost coincides with the theoretical noise level $\sigma_{N}$ (Fig. 11). The largest error of the $\sigma_{N E}$ calculation is obtained for the image \#28 «28_gravel», since its area is almost completely occupied with textures that are similar to noise (Fig. 10). To assess the accuracy of the proposed method, the root of mean square error (RMSE) was used, which was calculated between the values $\sigma_{N E}$ and $\sigma_{N}$ for all test images. 
In the mode \#1 of ROI selection (without image edges), a low RMSE error is obtained within the range of noise levels from $1 \%$ to $20 \%$ (Fig. 11a, Table 1), the error for all noise values $\mathrm{RMSE}=0.212$.

In the mode \#2 of ROI selection (taking into account the edges of image), the highest accuracy is reached for the threshold $T_{s}=0.08$ by edge detection in the image by the Sobel method (Fig. 11b, Table 1). The obtained error for all noise values RMSE $=0.210$, which is less than for the proposed method without taking into account the edges.

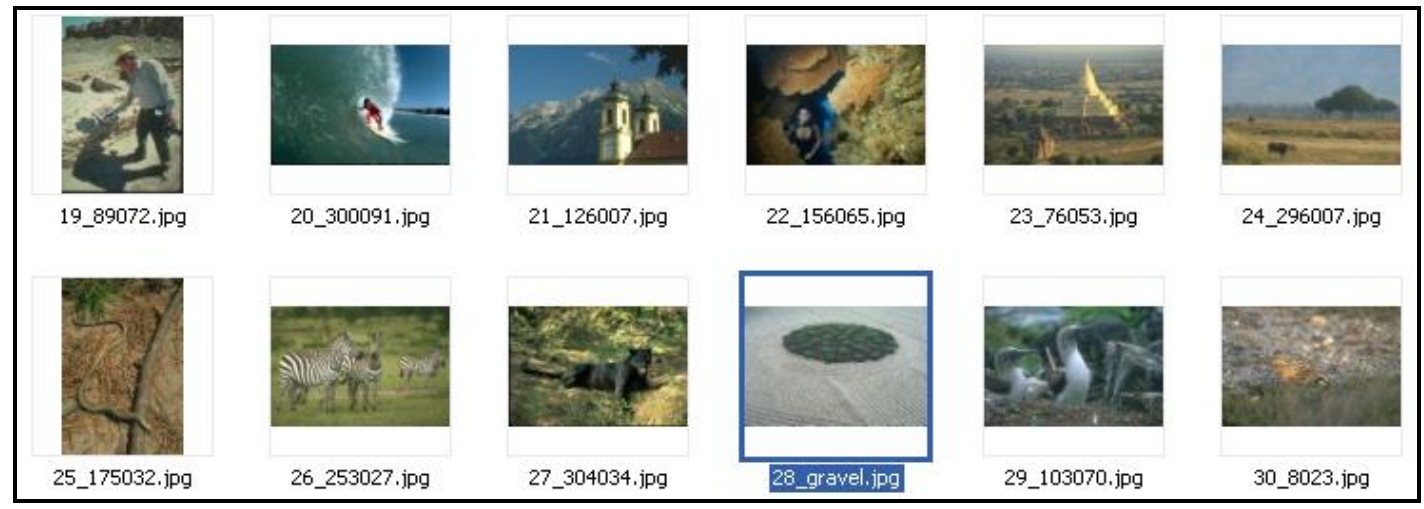

Fig.10. Test images of the Berkeley image database BSDS300 [13, 14], $481 \times 321$ pixels size, images \#19 - \#30 (from 100 images) is shown.

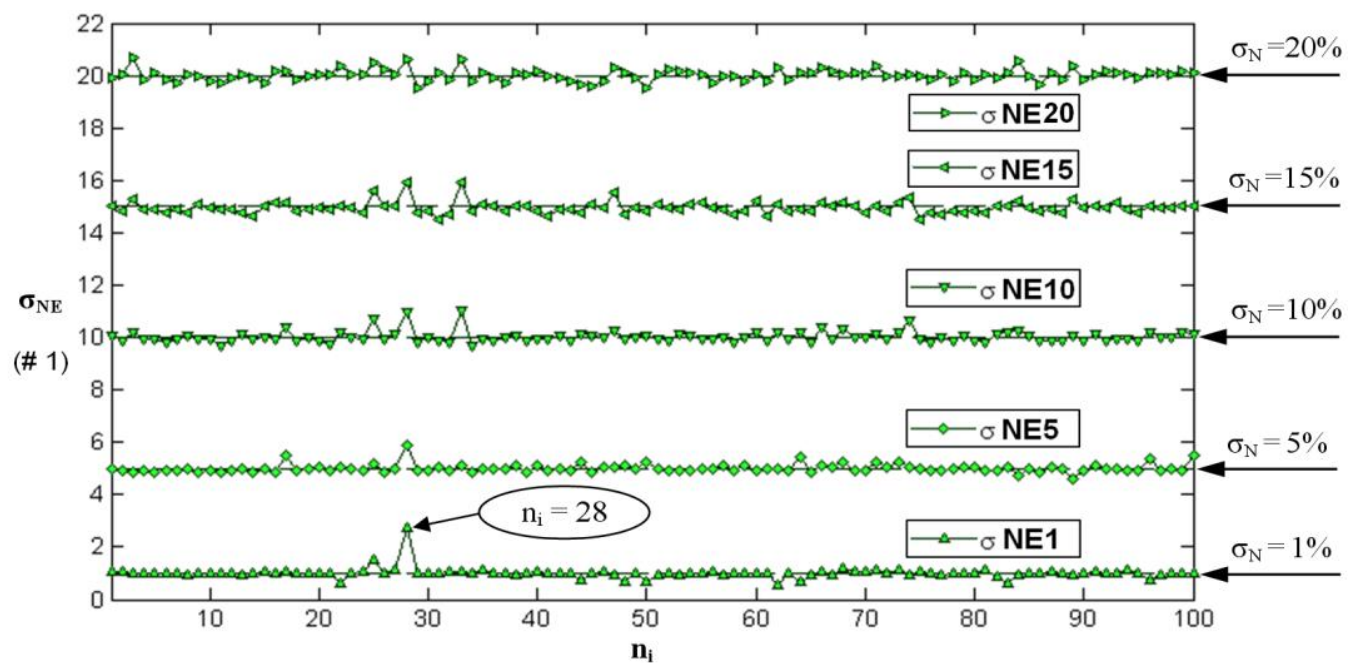

(a)

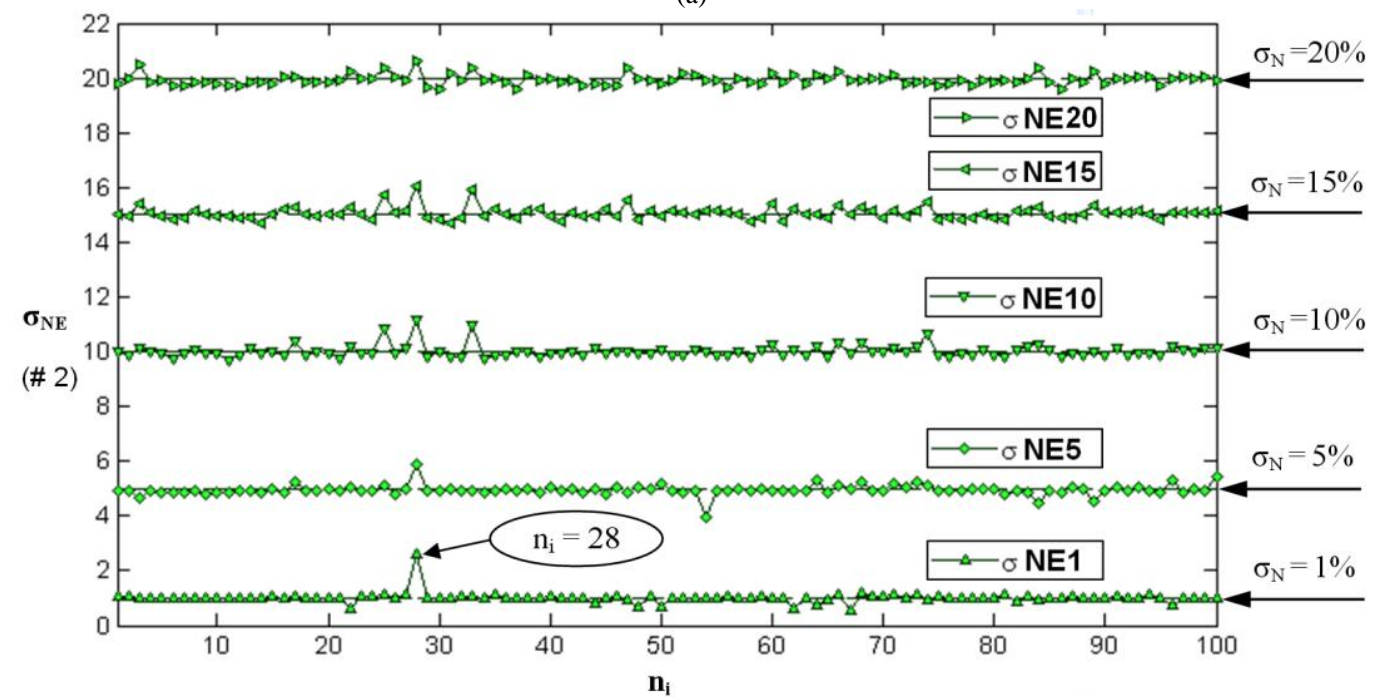

(b)

Fig.11. Results of the experimental Gaussian noise levels $\sigma_{N E}$ estimation by the proposed HLROI method for Berkeley image database BSDS300 (100 test images) [13, 14] with theoretical noise levels $\sigma_{N}(1 \%, 5 \%, 10 \%, 15 \%$ and $20 \%)$ : a) mode \#1 of ROI selection (without taking into account the edges of image); b) mode \#2 of ROI selection (taking into account the edges of image), threshold $T_{s}=0.08 ; n_{i}-$ number of image, $\sigma \mathrm{NE} 1, \sigma \mathrm{NE} 5$, $\sigma \mathrm{NE} 10, \sigma \mathrm{NE} 15, \sigma \mathrm{NE} 20$ - experimental values of noise levels for $\sigma_{N}=1 \%, \sigma_{N}=5 \%, \sigma_{N}=10 \%, \sigma_{N}=15 \%$ and $\sigma_{N}=20 \%$, respectively 
Thus, the results of the experimental Gaussian noise level estimation for the test images of the Berkeley image database BSDS300 (Table 2) show that the error of the proposed HLROI method (RMSE $=0.210)$ is noticeably lower than the statistical method error $($ RMSE $=1.686)$ and $0.067 \%$ less than the error of the PCAP method $(\mathrm{RMSE}=0.277)$.

Table 1. Results of experimental gaussian noise level $\sigma_{n e}(\%)$ estimation by proposed hlroi method (fig. 11) for 100 test images of base bsds300 $[13,14]$ with theoretical gaussian noise level $\sigma_{n} ; \sigma_{\text {nea }}-$ mean value of $\sigma_{n e} ; r_{m s e}$ - root mean square error, calculated between the values $\sigma_{n e}$ and $\sigma_{n}$ (for all images)

\begin{tabular}{|c|c|c|c|c|c|c|}
\hline $\begin{array}{r}\sigma_{N}, \\
\text { \% }\end{array}$ & $\begin{array}{c}\text { HLROI } \\
\text { into account } \\
\text { image the } \\
\text { edges) }\end{array}$ & $\begin{array}{c}\text { HLROI } \\
\text { (taking into } \\
\text { account the edges } \\
\text { of image, } \\
\text { threshold } \\
\text { Ts }=0.1 \text { ) }\end{array}$ & \multicolumn{2}{c|}{$\begin{array}{c}\text { HLROI } \\
\text { (taking into } \\
\text { account the edges } \\
\text { of image, } \\
\text { threshold } \\
\text { Ts }=0.08)\end{array}$} \\
\hline & $\sigma_{N E A}$ & $R_{M S E}$ & $\sigma_{N E A}$ & $R_{M S E}$ & $\sigma_{N E A}$ & $R_{M S E}$ \\
\hline 1 & 1.000 & 0.210 & 1.059 & 0.203 & 1.004 & 0.192 \\
\hline 5 & 5.003 & 0.164 & 4.978 & 0.218 & 4.950 & 0.197 \\
\hline 10 & 10.025 & 0.221 & 9.986 & 0.224 & 10.012 & 0.230 \\
\hline 15 & 14.957 & 0.236 & 15.054 & 0.220 & 15.062 & 0.229 \\
\hline 20 & 20.019 & 0.222 & 19.960 & 0.201 & 19.930 & 0.197 \\
\hline $\mathbf{1 . 2 0}$ & & $\mathbf{0 . 2 1 2}$ & & $\mathbf{0 . 2 1 3}$ & & $\mathbf{0 . 2 1 0}$ \\
\hline
\end{tabular}

Table 2. Results of experimental gaussian noise level $\sigma_{n e}(\%)$ estimation by statistical approach [6], method pcap [4] and proposed hlroi method (fig. 11b) for 100 test images of base bsds300 [13, 14] with theoretical gaussian noise level $\sigma_{n} ; \sigma_{\text {nea }}$ - mean value of $\sigma_{n e} ; r_{m s e}$ - rmse, calculated between the values $\sigma_{n e}$ and $\sigma_{n}$ (for all images)

\begin{tabular}{|c|c|c|c|c|c|c|}
\hline \multirow[t]{2}{*}{$\begin{array}{r}\sigma_{N} \\
\%\end{array}$} & \multicolumn{2}{|c|}{$\begin{array}{l}\text { Statistical } \\
\text { approach }\end{array}$} & \multicolumn{2}{|c|}{ PCAP } & \multicolumn{2}{|c|}{$\begin{array}{l}\text { Proposed method } \\
\text { HLROI (taking } \\
\text { into account the } \\
\text { edges of image, } \\
\text { threshold } \\
\text { Ts }=0.08 \text { ) }\end{array}$} \\
\hline & $\sigma_{N E A}$ & $R_{M S E}$ & $\sigma_{N E A}$ & $R_{M S E}$ & $\sigma_{N E A}$ & $R_{M S E}$ \\
\hline 1 & 2.151 & 2.011 & 1.068 & 0.304 & 1.004 & 0.192 \\
\hline 5 & 4.994 & 1.445 & 5.022 & 0.263 & 4.950 & 0.197 \\
\hline 10 & 9.737 & 1.61 & 10.052 & 0.264 & 10.012 & 0.230 \\
\hline 15 & 14.626 & 1.634 & 15.035 & 0.264 & 15.062 & 0.229 \\
\hline 20 & 19.559 & 1.678 & 20.009 & 0.288 & 19.930 & 0.197 \\
\hline $1 . .20$ & & 1.686 & & 0.277 & & 0.210 \\
\hline
\end{tabular}

When estimating the noise level for BSDS300 database of images [13, 14], the proposed method also showed a high convergence - an average of 6.4 iterations (excluding edges) and 5.5 iterations (including edges) are required for the selection of the ROI.

\section{Testing of Proposed Method For Photographs WITH REAL NOISE}

Using the proposed HLROI method and software (Fig. 12), the Gaussian noise level for a set of experimental images is calculated (Table 3). The average brightness of the scene for photos is calculated by the formula [17]:

$$
L_{B}=\frac{d_{p}^{2} \cdot K_{e}}{t_{e} \cdot I_{S O}},
$$

where $d_{p}-$ aperture; $K_{E}-$ reflected-light meter calibration constant $\left(K_{E}=12.5\right.$ for Canon and Nikon cameras); $t_{e}$ - exposure time; $I_{S O}-$ ISO.

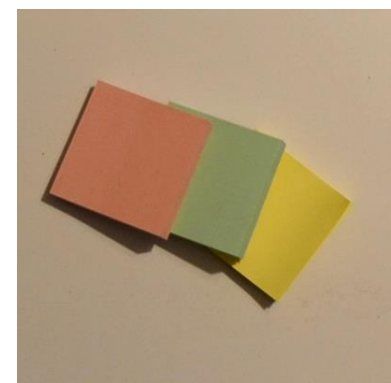

(a)

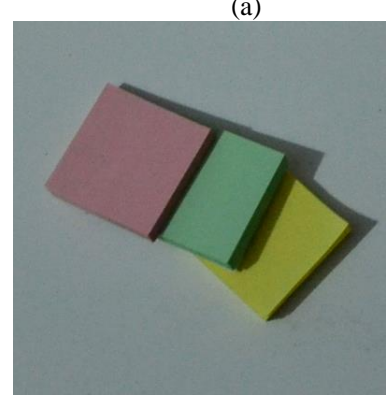

(c)

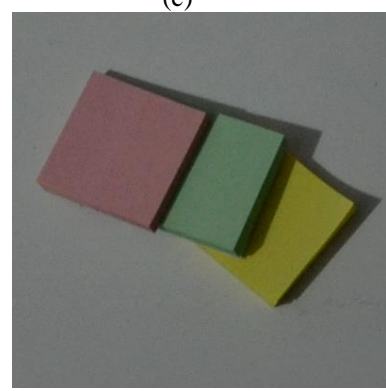

(e)

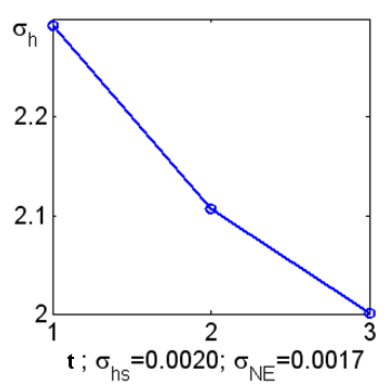

(b)

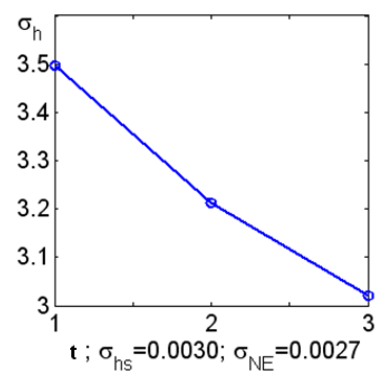

(d)

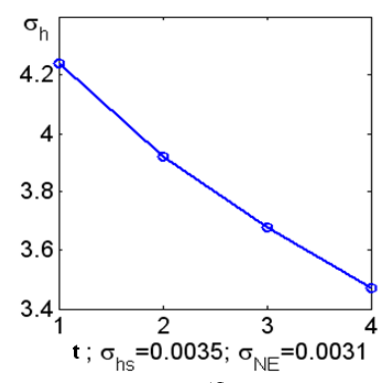

(f)
Fig.12. Examples of Gaussian noise level estimation by proposed method (without edges) in the image fragments (a), (c) (e) (images size $-400 \times 400$ pixels), obtained with camera "NIKON D3100" (Exposure time $t_{e}=1 / 15 \mathrm{c}$, aperture $d_{p}=3.5$ ), and graphs (b), (d), (f) of iterative refinement $\sigma_{h}$ : a) "Paper1.15,3.5,800" $\left(n_{i}=1\right)$; c) "Paper1.15,3.5,1600” ( $\left.n_{i}=2\right)$; e) Paper1.15,3.5,3200" $\left(n_{i}=3\right) ; n_{i}-$ number of image

Table 3. Gaussian noise levels $\sigma_{n e}$ estimated by the proposed method for experimental images (fig. 12)

\begin{tabular}{|c|c|c|c|}
\hline$n_{i}$ & $L_{B}, \mathrm{c}_{\mathrm{d}} / \mathrm{m}^{2}$ & ISO & $\sigma_{N E}, \%$ \\
\hline 1 & 2.871 & 800 & 0.17 \\
\hline 2 & 1.436 & 1600 & 0.27 \\
\hline 3 & 0.718 & 3200 & 0.31 \\
\hline
\end{tabular}

The test images (Fig. 12) are received at low light, so according to [1], Gaussian noise prevails in them. The obtained results (Table 3) show the dependence of the noise level on the lighting of the scene (the lower brightness of the scene corresponds to the higher noise level), which corresponds to the data $[17,18]$ and confirms the correctness of the method and the software developed to estimate the level of Gaussian noise. 


\section{CONCLUSION}

The proposed HLROI (High-Pass \& Low-pass Filtering \& Region Of Interest) method has been developed for high-precision automatic estimation of noise level in digital images, namely, standard deviation $\sigma_{N E}$ of Gaussian noise. The method consists in calculating the noise component of the image by means of high-pass filtration, selecting a ROI with a prevailing noise component using low-pass filtration, refining the ROI taking into account the image edges, calculating the histogram of the selected region and standard deviation $\sigma_{h}$ of the histogram, calculating the noise level $\sigma_{N E}$ based on the value $\sigma_{h}$.

The novelty of the investigation consists in getting an empirical formula (17) for calculating the threshold when selecting the ROI, empirical formulas (18-20) for calculating the experimental noise level, as well as using image edges (detected by Sobel method) to specify the ROI that provides the minimum error of noise level $\sigma_{N E}$ estimation.

The proposed method is implemented in MATLAB. The accuracy of the method has been verified in the processing of a set of 100 test images, where the root mean square error of the $\sigma_{N E}$ estimation is $0.210 \%$, which is $0.067 \%$ less than the error of the best analogous method PCAP. Taking into account the edges when calculating the ROI reduces the error of the noise level $\sigma_{N E}$ estimation for images with clear edges, the highest accuracy was achieved for the threshold $T_{s}=0.08$ of the Sobel method.

Noise level estimation for experimental photographic images by developed software gives results that correspond to the known data on the dependence of noise level in digital photos on the brightness of the scene.

The proposed method of noise level estimation can be used in graphic editors and video surveillance systems, as well as for educational purposes.

\section{REFERENCES}

[1] A. L. Bovik, The Essential Guide to Image Processing. Elsevier Inc., 2009.

[2] R. Gonzalez, R. Woods, Digital image processing, Prentice Hall, 2002.

[3] R. Gonzalez, R. Woods, L. Eddins, Digital Image Processing using MATLAB, Prentice Hall, 2004.

[4] X. Liu, M. Tanaka, M. Okutomi, "Single-Image Noise Level Estimation for Blind Denoising", IEEE Transactions on Image Processing, Vol. 22, No. 12, pp. 5226- 5237, Dec. 2013.

[5] S. Pyatykh, J. Hesser, L. Zheng, "Image noise level estimation by principal component analysis", IEEE Transaction on Image Processing, Vol. 22, No.2, pp. 687699, 2013.

[6] D. Zoran, Y. Weiss, "Scale invariance and noise in natural images", Proc. IEEE 12th Int. Conf. Comput. Vis., pp. 2209-2216, Sep./ Oct. 2009.

[7] W. Pratt, Digital Image Processing, John Wiley \& Sons, 1978.

[8] J. Immerkaer, "Fast Noise Variance Estimation", Computer Vision and Image Understanding, Vol. 64, No.
2, pp. 300-302, Sep. 1996.

[9] B. R. Corner, R. M. Narayanan, S. E. Reichenbach, "Noise estimation in remove sensing imagery using data masking”, Int. J. Remote Sensing, Vol. 24, No. 4, pp. 689$702,2003$.

[10] S.-C. Tai, S.-M. Yang, "A fast method for image noise estimation using Laplacian operator and adaptive edge detection", Proc. 3rd Int. Symp. Commun. Control Signal Process (ISCCSP), 12-14 March, Malta, pp. 1077-1081, 2008.

[11] G. Korn, T. Korn, Mathematical Handbook. For scientists and engineers, McGraw-Hill Book Company, 1968.

[12] Image Processing Place. Image Databases, 2017. http://www.imageprocessingplace.com/root_files_V3/ima ge_databases.htm.

[13] C. Fowlkes, D. Martin, J. Malik, "Local Figure/Ground Cues are Valid for Natural Images", Journal of Vision, Vol. 7 (8), No. 2, pp. 1-9, 2007

[14] The Berkeley Segmentation Dataset and Benchmark. BSDS300, 2017. https://www.eecs.berkeley.edu/Research/ Projects/ CS/vision/ bsds.

[15] Ye Zhiwei, Yang Juan, Zhang Xu, Hu Zhengbing, "Remote Sensing Textual Image Classification based on Ensemble Learning", International Journal of Image, Graphics and Signal Processing (IJIGSP), Vol. 8, No.12, pp.21-29, 2016.

[16] M. Srinivasa Rao, V.Vijaya Kumar, Mhm Krishna Prasad, "Texture Classification based on First Order Local Ternary Direction Patterns", International Journal of Image, Graphics and Signal Processing (IJIGSP), Vol. 9, No.2, pp. 46-54, 2017.

[17] ISO 2720:1974. Photography. General purpose photographic exposure meters (photoelectric type). Guide to product specification.

[18] D. Suresha, H. N. Prakash, "Data Content Weighing for Subjective versus Objective Picture Quality Assessment of Natural Pictures", International Journal of Image, Graphics and Signal Processing (IJIGSP), Vol. 9, No.2, pp. 27-36, 2017.

\section{Authors' Profiles}

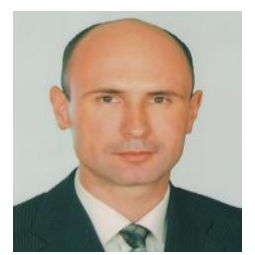

Serhiy V. Balovsyak, male, $\mathrm{PhD}$ of Physics and Mathematics Sciences (2003), Associate Professor of the Department of Computer Systems and Networks of Yuriy Fedkovych Chernivtsi National University, Ukraine.

Scientific interests are digital image processing, programming, image recognition, artificial neural networks, genetic algorithms.

Teaching interests are sensors, digital signal processing, artificial intelligence, user interfaces.

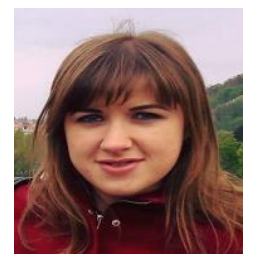

Khrystyna S. Odaiska, female, $\mathrm{PhD}$ Student of the Department of Computer Systems and Networks of Yuriy Fedkovych Chernivtsi National University, Ukraine.

Scientific interests are digital image processing, programming, image recognition.

Teaching interests are data base, programming, computer graphics. 
How to cite this paper: Serhiy V. Balovsyak, Khrystyna S. Odaiska," Automatic Highly Accurate Estimation of Gaussian Noise Level in Digital Images Using Filtration and Edges Detection Methods", International Journal of Image, Graphics and Signal Processing(IJIGSP), Vol.9, No.12, pp. 1-11, 2017.DOI: 10.5815/ijigsp.2017.12.01 